\title{
MyopicMAC: A Throughput-Optimal Random Access Scheme for Distributed Wireless Networks
}

\author{
Rodolfo Oliveira $^{1,2} \cdot$ Miguel Luis $^{1,2} \cdot$ Luis Bernardo ${ }^{1,2}$ • \\ Rui Dinis ${ }^{1,2}$
}

Published online: 14 August 2015

(C) Springer Science+Business Media New York 2015

\begin{abstract}
This work presents a novel Medium Access Control (MAC) algorithm for single-hop distributed wireless networks, MyopicMAC, which is designed to maximize the network throughput performance. In our scheme, all nodes adopt a common optimal contention window avoiding message passing, which is obtained through the use of the common view of the channel. MyopicMAC is also designed to improve the medium access fairness. This goal is achieved by adopting a contention window similar for all nodes. Departing from the optimal throughput for a saturated network, we devise a scheme to estimate the number of nodes, which is a prime parameter to regulate the medium access control. It is shown that each node is able to estimate the number of competing nodes by using both its own medium access probability and the idle slot probability observed in the channel. Several simulation results evaluate the throughput, fairness and delay performance obtained with our proposal. The possibility of transmission errors is also taken into account. A comparison is made with several state-of-the-art MAC algorithms, different number of competing nodes and different behaviors expressed by different transmission error probabilities. The obtained results indicate that MyopicMAC performs closer to the optimal throughput and simultaneously exhibit high medium access fairness. Moreover, because MyopicMAC does not take the success of previous transmissions in the contention regulation, the performance of the MAC algorithm is not affected by transmission errors.
\end{abstract}

Keywords Medium access control - Wireless LANs - MAC performance evaluation · MAC optimization

Miguel Luis

nmal@campus.fct.unl.pt

Rodolfo Oliveira

rado@fct.unl.pt

1 Departamento de Engenharia Electrotécnica, Faculdade de Ciências e Tecnologia, FCT, Universidade Nova de Lisboa, 2829-516 Caparica, Portugal

2 Instituto de Telecomunicações-IT, Av. Rovisco Pais, 1, 1049-001 Lisboa, Portugal 


\section{Introduction}

In the last years, there has been an increasing interest in developing and testing optimal Medium Access Control (MAC) algorithms for ad hoc networks. The wireless ad hoc networks are characterized by the lack of an infrastructure able to centralize the node's MAC scheme. Although the lack of infrastructure is an obvious advantage, because it allows fast and costless network deployment, it also increases the complexity of the MAC algorithms: if multiple nodes simultaneously access the channel, their mutual interference corrupts both transmissions, which are unsuccessful. A MAC algorithm should allow channel access to every node in an efficient way. This means that MAC algorithms should be designed to maximize the throughput, minimize the medium access delay and allow the nodes to access the channel in a fair way. While these objectives are well identified, it is not obvious if there exists a random access algorithm that is both throughput and delay optimal [1].

This work presents a novel MAC scheme, MyopicMAC, which is designed to maximize the network throughput performance of a distributed single-hop network. It follows the idea of dynamically adapting the medium access probability, which was already addressed in several works such as [2-7]. In MyopicMAC each node estimates the channel utilization (measuring the number of idle and busy slots) as well as the number of nodes competing for the channel. These parameters are obtained only by channel observations and, because all nodes have a shared knowledge of it, no message passing is needed. While other works such as $[3,4,8]$ also propose solutions where no message passing is needed, our proposal uses a new optimization principle which does not rely in observed approximations, such as the one adopted in [3] and [4]. Departing from the optimal throughput for a saturated network, we devise a scheme to estimate the number of nodes, which is a prime parameter to regulate the medium access contention window. The number of nodes is estimated assuming that all competing nodes are operating near the steady state. This assumption simplifies the estimation process, since the medium access probability of the node performing the estimation is similar to the medium access probability of its neighbors. Thus, a node can use its own medium access probability in the estimation process as an approximate value of the probability of its neighbors.

The main contributions of our approach are as follows:

- MyopicMAC is based on a novel distributed and optimal-throughput contention window regulation scheme. This scheme is designed to avoid message passing: only channel information is used, which is sampled by each node;

- MyopicMAC exhibits high performance in terms of medium access fairness. Since the all nodes adopt approximately the same contention window, the access fairness measured with the Jain's index [9] exhibits high levels of fairness;

- Contrarily to other MAC protocols, such as IEEE 802.11, where transmission errors increase the contention period because the contention window regulation takes the success of the previous transmission attempt in the window regulation process, MyopicMAC's contention window regulation does not depend on the success of the previous attempt. This means that MyopicMAC's performance is not affected by transmission errors.

A characterization of MyopicMAC's performance is also reported. We present an extensive comparison of multiple simulation results in a Wireless Local-Area Network (WLAN) scenario to evaluate the performance of our proposal with several state-of-the-art 
MAC algorithms. The results indicate that the proposed solution achieves our initial motivation goals, since it exhibits high degree of medium access fairness and its throughput is close to the optimal one. The results also show that MyopicMAC throughput is not affected by transmission errors.

The rest of this paper is organized as follows. The next section provides an overview of related work. Section 3 introduces the considered system. MyopicMAC algorithm and its implementation are described in Sects. 4 and 5, respectively. Simulation results shown in Sect. 6 compare the performance of our approach with other MAC algorithms. Concluding remarks are given in Sect. 7.

\section{Related Work}

Four decades have elapsed since the publication of Abramson's Aloha system [10]. In the interim we can cathegorize the proposed MAC algorithms into two types [11]: Random Access (RA) algorithms and Scheduling-Based (SB) algorithms. While centralised SB algorithms are contention-free (e.g. TDMA), RA algorithms are contention-based (e.g. slotted Aloha). However, in the last years several attempts have been made to design Distributed SB (DSB) algorithms for throughput maximization, which take the form of random access algorithms [12].

DSB algorithms (e.g. [3, 12-14]) are usually based on medium access utility functions [15], which describe the individual profit of each node for accessing the channel. These algorithms are frequently derived from an optimization problem, which is generally referred to as a Network Utility Maximization (NUM) problem (for a related survey, see [16]). NUM problems try to maximize the utility functions constrained by several conditions. These conditions may impose specific access fairness policies, medium access probability bounds, algorithm's stability thresholds, etc.. The most representative DSB algorithms are the throughput optimal-based ones. These algorithms are commonly based on the Maximum Weight (MW) algorithm proposed by Tassiulas and Ephremides [17], which is known to provide optimal throughput in the network's stable region of operation. MW algorithm is centralized, which means that a given node needs to have knowledge of the properties of all neighbors competing for the medium. Several randomized MW-based algorithms have been proposed in order to decentralize it [18, 19]. However they still require a significant amount of message passing to solve each iteration of the algorithm. Other DSB algorithms, such as [14] and [3], do not follow the MW principle. Instead, they use different utility functions to formulate a NUM problem. However, most of times the NUM formulation adopts utility functions and/or constraints that do not comply with several requirements needed to apply multi-criterion optimization techniques, provided by existing optimization theories [20]. To solve this class of problems, the optimal solution is then relaxed into an implementable algorithm. This is the case for [11] and [14], where the relaxation process decreases the final optimization efficiency, as several approximations are used to translate the optimal solution into a practical algorithm. Moreover, these algorithms require extensive message passing among the nodes, and consequently wasting a significant amount of throughput. An exception is the work in [3], which avoids message passing. But the authors only prove that the algorithm converges for a large number of nodes and consider a few non-realistic assumptions such as infinite backlogs and frequent message passing among the nodes, which is a requirement to estimate the packet error rate of the communication channel. 
RA algorithms (e.g. [4, 8, 21-24]) usually adopt slotted time-domain and unit-sized packets in a common control channel. These algorithms use successful transmissions or busyness of the channel as an indirect measure of the number of competing nodes, which is employed to regulate the randomized medium access of each node. RA algorithms can be based on simple heuristics. One of the most popular heuristic RA algorithm is the exponential backoff adopted in the IEEE 802.11 standard [21], which is based on the Carrier Sense Multiple Access with Collision Avoidance (CSMA/CA) mechanism. From the huge amount of heuristic RA algorithms described in the literature [22], we highlight the "Gentle Distributed Coordination Function" (GDCF) [23], because of its performance in terms of throughput. While in IEEE 802.11 a node sharply cuts the contention window after a successful transmission and independently of the number of nodes accessing the channel, GDCF adopts a heuristic that incorporates a more conservative approach: a node does not change its contention window after a successful transmission. Moreover, if a node does eight consecutive successful transmissions, its contention window is halved. The contention window is doubled after a failed transmission (when the ACK frame is not received). Other sub-class of RA algorithms adopt optimal contention window regulation inspired in prior behaviors of specific random access schemes. These include the well known "Assymptotically-Optimal Backoff" (AOB) [8] and "Idle Sense" (IS) [4] algorithms.

AOB optimizes the IEEE 802.11 throughput and assumes that the optimal medium access is almost independent of the number of active stations. This is a rough approximation, namely for a small number of nodes, but it achieves close to optimal throughput when the number of nodes increase above 10. Basically, each node adopts the exponential backoff regulation used in IEEE 802.11. But contrarily to IEEE 802.11, a node does not necessarily access the channel when the backoff counter reaches zero. The effective channel access depends on the probability of successful transmission, evaluated at the end of each contention period. If the probability is high, a node does the channel access. Otherwise, the transmission is rescheduled as a collision would have occurred, i.e., the node doubles the contention window and enters in a new contention period. A frame involved in a transmission error can be retransmitted up to a defined maximum number of times, and the contention window regulation follows the one proposed in the IEEE 802.11 algorithm.

IS [4] tries to adopt the node's access behavior that maximizes the throughput by minimizing the time wasted in contention and collisions, and thus increasing the available time for transmissions. IS operation relies in the observation that the optimal throughput is maximized when approximately 5.68 [4] consecutive idle slots are sensed, and this value almost does not depend on the number of nodes competing for the medium. The contention window used to retransmit frames involved in transmission errors or collisions is determined during the contention period of the failed attempt. Basically the contention window is regulated in order to meet the mentioned average number of consecutive idle slots (5.68) during the contention period.

The majority of DSB algorithms are relatively easier to analyze but they are inherently poor in performance because of their requirements in terms of frequent message passing and/or oversimplification (relaxation) of the optimal solution [25]. RA algorithms are harder to analyze, but because they present an easier implementation that most of the times avoids message passing, they can achieve better throughput [25]. The algorithm presented in this work lies in the RA algorithms class. 


\section{System Description}

We consider a single-hop network represented by the set $\mathcal{N}$ of $n$ nodes. A successful transmission implies that only one node transmits to the shared medium. When two or more nodes simultaneously access the channel, their mutual interference corrupts both transmissions, which are unsuccessful. All nodes are traffic-saturated i.e., a node always has a frame to transmit. When a node has a new frame to transmit, it senses the channel activity. If the channel is sensed idle for a given period of time (Distributed Inter-Frame Space (DIFS) [21]) the node transmits. Otherwise, if the channel is sensed busy, the node starts a random contention period to minimize the frame collision probability. In the contention period a node starts to charge a Contention Counter with the number of slots randomly chosen according to a uniform distribution in the range $(0, W-1]$, where $W$ is the Contention Window. The contention counter is decremented as long as the channel is sensed idle, paused when a transmission is detected, and resumed when the channel is sensed idle again (after a transmission) for a period equal to a DIFS interval. The transmission only occurs when the backoff counter reaches zero. We consider that idle slots have a shorter duration than busy slots following the same rationale and timings defined in IEEE $802.11^{1}$ [21].

The behavior described above is similar with IEEE 802.11 standard. But contrarily to IEEE 802.11, where the contention window is doubled when a frame collides, we consider an optimal throughput MAC scheme where the nodes use a contention window that is close to the optimal one (optimal in the sense of maximizing the throughput), and is regulated at the end of each transmission attempt. By this way, the network nodes always use a single contention window that does not depend on the previous transmission state (collision or success) as is the case with IEEE 802.11 exponential backoff.

\subsection{Theoretical Throughput}

Let us define $\tau$ as the probability that a node transmits in a randomly chosen slot time. Because the contention window regulation does not depend on previous transmission state, we adopt a given contention window, $W$, for each transmission attempt. This case is similar to IEEE 802.11 contention mechanism when a single backoff stage is used. Considering that the medium access decisions are independent and identically distributed, the saturated stationary access probability $\tau$ follows the Eq. (8) in [26], which states that

$$
\tau=\frac{2}{W+1} .
$$

Let $S$ be the normalized system throughput. To compute $S$ we must describe the probability of finding slots where the channel may be found idle, with a probability

$$
p_{i}=(1-\tau)^{n}
$$

or busy due to a single transmission with the probability

\footnotetext{
${ }^{1}$ IEEE 802.11 adopts slots with constant duration when the channel is idle (idle slots), which are always shorter than the duration of a single (or multiple) transmission(s) (busy slot).
} 


$$
p_{s}=n \tau(1-\tau)^{(n-1)}
$$

or busy due to multiple transmissions (collisions) with probability

$$
p_{c}=1-p_{s}-p_{i}
$$

The system throughput follows the classical definition for contention-based MAC schemes (e.g. [26, 27]), and is expressed as the ratio of the amount of time that the channel is successfully used, to the expected contention duration:

$$
S=\frac{p_{s}\left(1-p_{e}\right) \mathbb{E}\left[L_{D A T A} / R\right]}{p_{i} \mathbb{E}\left[T_{i}\right]+p_{s} \mathbb{E}\left[T_{s}\right]+p_{c} \mathbb{E}\left[T_{c}\right]},
$$

where $\mathbb{E}\left[T_{s}\right], \mathbb{E}\left[T_{c}\right]$ and $\mathbb{E}\left[T_{i}\right]$ denote the expected duration of a successful transmission, collision and idle slot, respectively. $\mathbb{E}\left[L_{D A T A} / R\right]$ denotes the expected frame payload duration, where $L_{D A T A}$ is the frame payload length and $R$ is the transmission rate. The probability of transmission error is $p_{e}$.

Note that $S$ represents the amount of channel capacity successfully used when the network operates at the stationary regime. This means that we have assumed that all nodes adopt the same stationary access probability $\tau$, and because all nodes access with the same chance, the throughput definition expressed in (5) considers a MAC scheme that is said to be fair.

\subsection{Transmission Errors}

To assume a more realistic transmission system, we adopted the IEEE 802.11 Directed Sequence Spread Spectrum transmission modulations specified for $11 \mathrm{Mbps}$ data transmission rate. Basically, a frame is formed by a PHY preamble that uses the DBPSK modulation (Differential Binary Phase Shift Keying), and a MAC header and data payload that uses CCK modulation (Complementary Code Keying). Assuming different probabilities of bit error for DBPSK and CCK modulations, $p_{b_{1}}$ and $p_{b_{2}}$ respectively, the probability of transmission error [also designated as Frame Error Ratio (FER)] is given by

$$
p_{e}=1-\left(1-p_{b_{1}}\right)^{L_{P H Y}}\left(1-p_{b_{2}}\right)^{L_{M A C}+L_{D A T A}},
$$

where $L_{P H Y}$ is the length of the PHY preamble and $L_{M A C}+L_{D A T A}$ is the length of the MAC header plus the frame's data payload, respectively. Considering an additive white Gaussian channel, the bit error probabilities for the considered modulations are given by [28]

$$
p_{b_{1}}=\frac{1}{2} e^{\left(-\frac{E_{b}}{N_{0}}\right)},
$$

and,

$$
p_{b_{2}}=1-\frac{1}{\sqrt{2 \pi}} \int_{-y}^{\infty}\left(\frac{1}{\sqrt{2 \pi}} \int_{-(v+y)}^{(v+y)} e^{-\frac{x^{2}}{2}} d x\right)^{\frac{M}{2}-1} e^{-\frac{v^{2}}{2}} d v
$$

where $E_{b} / N_{0}$ is the signal-to-noise ratio per bit, $y=\sqrt{E_{b} / N_{0}}$, and $M=8$ bits per symbol. 


\section{MyopicMAC Access Scheme}

\subsection{Optimal Medium Access Probability}

Departing from the throughput derived in (5), and considering that the expected durations are constants i.e, $\mathbb{E}\left[L_{D A T A} / R\right]=D, \mathbb{E}\left[T_{s}\right]=T_{s}, \mathbb{E}\left[T_{i}\right]=T_{i}, \mathbb{E}\left[T_{c}\right]=T_{c}$, which is a good approximation even using simple estimators as the one proposed for $T_{s}$ in [8], $S$ can be rewritten as

$$
S=\left(1-p_{e}\right) \frac{D}{T_{s}-T_{c}+\frac{T_{c}+p_{i}\left(T_{i}-T_{c}\right)}{p_{s}}} .
$$

To maximize $S$, we note that the probabilities of transmission error $\left(p_{e}\right)$ and medium access $(\tau)$ are independent, because the contention window regulation does not depend on the success of the previous attempts. Since the error probability $p_{e}$ is an intrinsic feature of the physical layer, $S$ can only be maximized by finding the optimal access probability $\tau^{\star}$ that solves the following equation

$$
\left(1-\tau^{\star}\right)^{n}\left(T_{c}-T_{i}\right)+\left(n \tau^{\star}-1\right) T_{c}=0
$$

(full details of this result are presented in the Appendix). The optimal throughput ${ }^{2} S^{\star}$ is achieved by imposing to each node the access probability $\tau^{\star}$, which can be written as a function $f$ of the number of nodes

$$
\tau^{\star}=f(n),
$$

because $T_{c}$ and $T_{i}$ are constants. In this way, the optimal medium access probability $\tau^{\star}$ only depends on the number of nodes competing for the medium and $f(n)$ is a decreasing function for $n>0$. Equation (11) stresses the importance of having an estimate of the number of nodes, because if the number of nodes is known we can solve (10) to find the optimal medium access probability $\tau^{\star}$.

\subsection{Estimation of the Number of Nodes}

A node can measure the probability of finding an idle slot, denoted by $\tilde{p}_{a}$, by observing the state of the slots during the period of contention. To keep $\tilde{p}_{a}$ in a slowly time varying value, and consequently close to its steady state, we filter the slot samples as is detailed in the next section. If the network is operating near a stable point, all nodes adopt the medium access probability $\tau^{\star}$. When this occurs, a node listens to an idle slot if the remaining $n-1$ nodes do not transmit, meaning that $p_{a}=\left(1-\tau^{\star}\right)^{(n-1)}$. By this way, the number of nodes can be estimated by minimizing the squared error between the measured probability $\left(\tilde{p}_{a}\right)$ and the optimal one $\left(p_{a} \approx\left(1-\tau_{i}\right)^{(n-1)}\right.$, where $\tau_{i}$ represents the individual medium access probability of each neighbor node). The estimated number of nodes $\hat{n}$ can be approximated by

\footnotetext{
${ }^{2}$ Since all nodes access with the optimal medium access probability $\tau^{\star}$, the optimal throughput $S^{\star}$ represents the optimal-fair throughput.
} 


$$
\hat{n}=\underset{n \in \mathcal{N}}{\operatorname{argmin}} \sqrt{\left(\tilde{p}_{a}-\left(1-\tau_{i}\right)^{n-1}\right)^{2}} .
$$

However, there is an open question: the nodes do not know what is the stable point of operation, and $\tau_{i}$ in (12) is unknown. Since all nodes will adopt similar contention windows, in the steady state a node $n_{y}$ can use its own probability of medium access to estimate the number of nodes $\left(\tau_{i}=\tau_{y}\right)$. If the network is operating far from the stable point, the accuracy of the estimates decrease and this approach is only valid if $\tau_{y}$ converges to the stationary optimal point of operation $\tau^{\star}$. Thus we must consider the following assumption:

$\mathcal{A}$ - It is possible to design a MAC scheme where a node $n_{y}$ uses its own medium access probability $\tau_{i}=\tau_{y}$ in (12) to determine the optimal access probability ( $\tau^{\star}$ given by (11)) and simultaneously guarantee that its medium access probability converges to the optimal value.

This assumption is investigated in the next subsection.

\subsection{Contention Window Regulation}

We start to consider the medium access probabilities vector $\mathbf{T}(\mathbf{k})=\left(\tau_{\mathbf{1}}(\mathbf{k}), \tau_{\mathbf{2}}(\mathbf{k}), \ldots\right.$, $\left.\tau_{n}(k)\right)$, which describes the probabilities applied by each node in the contention period $k \in\{0,1, \ldots, m\}$. Since the contention window only assumes integer values, the individual access probabilities $\tau_{1}(k), \tau_{2}(k), \ldots, \tau_{n}(k)$ only take values in a finite set represented by $\mathcal{T}=\left\{2 /\left(W_{\max }+1\right), 2 /\left(W_{\max }\right), 2 /\left(W_{\max }-1\right), 2 /\left(W_{\max }-2\right), \ldots, 2 /\left(W_{\min }+1\right)\right\}, \quad$ where $W_{\min }>0$ and $W_{\max }>W_{\min }$ are the minimum and maximum adopted contention window values, respectively. Because $\mathcal{T}$ is finite, $\mathbf{T}$ is a countable Markov chain that describes all possible medium access probability values adopted by all network nodes.

A long and fixed contention period $(k=0)$ is initially used by each node, and its purpose is to initialize the $\tilde{p}_{a}$ estimator. The nodes only access the channel when the contention period for $k=1$ finishes. The contention window applied in the period $k=1$ jointly with the initialization done in $k=0$ guarantees that each node has a first measure of $\tilde{p}_{a}$ at the end of the slot $k=1$. The vector $\mathbf{T}$ is then initialized with a given probability $0<\tau_{\text {init }}<1, \tau_{1}(1)=\tau_{2}(1)=\ldots=\tau_{n}(1)=\tau_{\text {init }}$, and all nodes use the first contention window given by $W_{\text {init }}=2 / \tau_{\text {init }}-1$. After this, a node starts to adapt the medium access probability each time the contention counter reaches zero. The adaptation process is illustrated in Fig. 1. When the contention period $k>0$ finishes, a node uses the expression (12) with the measure $\tilde{p}_{a}$ and $\tau_{i}=\tau_{\text {init }}$ to obtain the estimate for the number of competing nodes $(\hat{n}(k)$ ). Then $\hat{n}(k)$ is used as an argument in (11) to obtain a reference for the medium access probability $\tilde{\tau}(k)$, which is later imposed by the controller $C(\tilde{\tau}(k))$. The controller output $\tau(k+1)$ is then used to define the contention window

$$
W(k+1)=\frac{2}{\tau(k+1)}-1
$$

adopted in the next contention period. Note that for future contentions periods of node $n_{y}$ at $k+1=2,3, \ldots, m$, the number of nodes $\hat{n}(k)$ is obtained using the Eq. (12), where $\tau_{i}$ is

Fig. 1 Block diagram for the MAC scheme

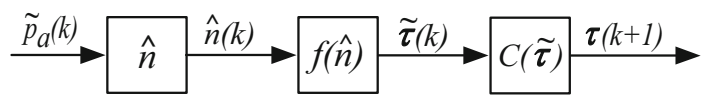


replaced by the medium access probability felt in the finished contention period $k$ : $\tau_{i}(k=2)=\tau_{y}(k=1), \tau_{i}(k=3)=\tau_{y}(k=2), \ldots, \tau_{i}(k=m)=\tau_{y}(k=m-1)$.

To test the assumption $\mathcal{A}$ we employ the technique of Lyapunov drift, which has been successfully used to stabilize several systems, such as packet switch systems (e.g. [29]). This allows us to deduce the stability-in-the-mean of the system, and because the MAC scheme is markovian (the contention window for the future instant $k+1$ depend only upon the present instant $k$ ), such stability is equivalent to the existence of a steady state distribution.

Using a quadratic Lyapunov function $L(\mathbf{T})=\sum_{\mathbf{i}=\mathbf{1}}^{\mathbf{n}} \tau_{\mathbf{i}}^{\mathbf{2}}$, and assuming that $\mathbf{T}(\mathbf{k})$ evolves according to some probabilistic law, the MAC scheme is strongly stable if there exists constants $B>0$ and $\epsilon>0$, such that for all time instants $k$ we have [30]

$$
\mathbb{E}\{L(\mathbf{T}(\mathbf{k}+\mathbf{1}))-\mathbf{L}(\mathbf{T}(\mathbf{k})) \mid \mathbf{T}(\mathbf{k})\} \leq \mathbf{B}-\epsilon \sum_{\mathbf{i}=\mathbf{1}}^{\mathbf{n}} \tau_{\mathbf{i}}(\mathbf{k}) .
$$

The condition (13) represents the Lyapunov Stability criterion and when it holds, for any $\delta>0$, the Lyapunov drift, denoted by $\Delta(\mathbf{T}(\mathbf{k})) \triangleq \mathbb{E}\{\mathbf{L}(\mathbf{T}(\mathbf{k}+\mathbf{1}))-\mathbf{L}(\mathbf{T}(\mathbf{k})) \mid \mathbf{T}(\mathbf{k})\}$, satisfies $\Delta(\mathbf{T}(\mathbf{k})) \leq-\delta$ whenever $\sum_{i=1}^{n} \tau_{i}(k) \geq(B+\delta) / \epsilon$. In other words, the condition (13) ensures that the Lyapunov drift is negative whenever the sum of the medium access probabilities is sufficiently large. Intuitively, this property ensures MAC scheme stability because, whenever the vector $\mathbf{T}$ leaves the bounded region $\left\{\mathbf{T} \geq \mathbf{0} \mid \sum_{i=1}^{n}\right.$ $\left.\tau_{i}(k) \leq(B+\delta) / \epsilon\right\}$, the negative drift eventually drives it back to this region [30].

To test the stability of our MAC scheme, and consequently the validity of the assumption $\mathcal{A}$, we study the Lyapunov drift for two different cases: (1) increasing the number of network nodes; (2) decreasing the number of network nodes. These cases are representative of the network changes where our MAC scheme must guarantee convergence to the steady state optimal medium access probability.

\subsubsection{The Number of Network Nodes Increases}

Considering that at instant $k-1$ the network is operating with $K_{e}$ nodes, $n(k-1)=K_{e}$, and, during the contention period $k, K_{a}$ nodes are added to the network, such that $n(k)=K_{e}+K_{a}$. Admitting that during the contention period $k-1$ the contention regulation was close to the steady state optimal point of operation, when the aditional $K_{a}$ nodes access the medium in the slot $k$, they will decrease the measured probability $\tilde{p}_{a}$ used in (12). Since $K_{e}$ nodes use their current medium access probability $\tau(k)$ to determine $\tau(k+1)$, from (12) we conclude that if $\tilde{p}_{a}(k)<\tilde{p}_{a}(k-1)$ then $\hat{n}(k)>\hat{n}(k-1)$. Since the decreasing function $f(n)$ is used to determine $\tau(k+1)$, then $\tau(k+1)<\tau(k)$. In this way, the Lyapunov drift is negative, meaning that the existing nodes will successfully decrease their medium access probability to adapt to node's increase.

\subsubsection{The Number of Network Nodes Decreases}

In the same way, we consider that at instant $k-1$ the network is operating with $n=K_{e}$ nodes and, during the instant $k, K_{d}<K_{e}$ nodes stop transmitting, such that $n=K_{e}-K_{d}$. Admitting that the existing $K_{e}$ nodes are close to the steady state optimal point of operation, when $K_{d}$ nodes stop transmitting they will increase the measured probability $\tilde{p}_{a}$ used in (12). Because the remaining $K_{e}-K_{d}$ nodes use their current medium access probability 
$\tau(k)$ to determine $\tau(k+1)$, from (12) we conclude that if $\tilde{p}_{a}(k)>\tilde{p}_{a}(k-1)$ then each node observes that $\hat{n}(k)<\hat{n}(k-1)$. Since the decreasing function $f(n)$ is used to determine $\tau(k+1)$, then $\tau(k+1)>\tau(k)$. For future steps $k+2, k+3, \ldots, k+G$ the nodes will increase their medium access probability, while $\tilde{p}_{a}(k+2)>\tilde{p}_{a}(k+1), \ldots, \tilde{p}_{a}$ $(k+G)>\tilde{p}_{a}(k+G-1)$, because the filter used to sample $\tilde{p}_{a}$ introduces a delay. But after $G+1$ periods, the measure $\tilde{p}_{a}(k+G+1)<\tilde{p}_{a}(k+G)$, since the nodes are increasing their medium access probability. When this happens, the condition $\hat{n}(k+G+1)>\hat{n}(k+G)$ holds, and consequently, the transmission probability for the instant $(k+G+2)$ will guarantee a negative drift that stabilizes our MAC scheme: $\tau(k+G+2)<\tau(k+G+1)$.

\section{MyopicMAC Implementation Details}

MyopicMAC is designed to maximize the network throughput and increase the channel access fairness. These improvements are achieved because all nodes regulate their contention windows in order to be close to the optimal medium access probability. As depicted in Fig. 1, the optimal access probability can be computed in four stages: 1) idle slot probability measurement, 2) estimation of the number of nodes, 3) optimal access probability computation and 4) access probability control. Stages 1) and 4) are detailed next.

As mentioned in Sect. 4.2, the idle slot probability can be measured by observing the state of the slots during the period of contention. We define the following relative frequency

$$
f_{s}=\frac{1}{B} \sum_{i=1}^{B} \text { Slot }_{i},
$$

where $S$ Sot $_{i}$ is equal to 0 if the $i$-th contention slot is sensed idle, or equal to 1 if during the $i$-th slot the channel is sensed busy. $B$ is the number of observed slots. Note that similarly to

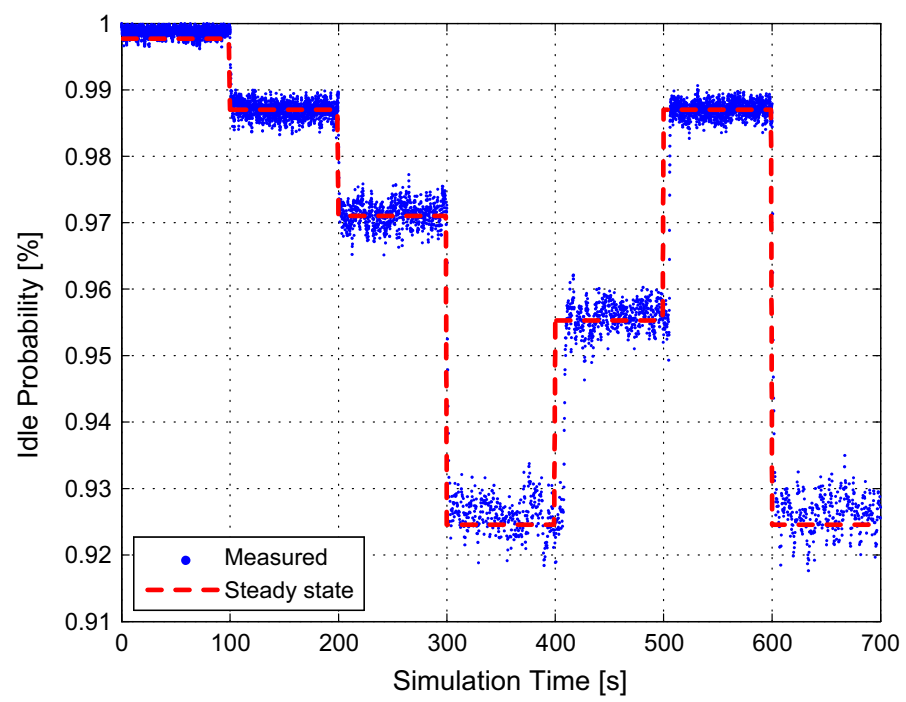

Fig. 2 Idle slot probability for the case when the nodes use a fixed contention window 
IEEE 802.11, in the proposed scheme each node must sense the channel in each slot because busy and idle slots have different durations. Thus $f_{s}$ can be obtained in a costless way because nodes already sense each slot to implement the contention waiting time.

To obtain a slowly-varying measure of $\tilde{p}_{a}$ we used an Auto Regressive Moving Average (ARMA) filter. This filter uses $f_{s}$ in (14) and the slowly-varying measure is given by

$$
\tilde{p}_{a}(k)=\alpha \tilde{p}_{a}(k-1)+(1-\alpha) f_{s},
$$

where $\alpha$ represents the filter memory. Note that $f_{s}$ takes the last $B$ slot samples into account. But because idle slots have a shorter duration than busy slots, this means that $B$ slots can represent a short period of time if its average duration is close to an idle slot, or a longer period of time if the slot's average duration increases. This means that the time needed to sample $B$ slots is not linear with the number of slots, since it also depends on the slots' busyness. We term this issue as "non-linear slot busyness sampling", which is also reported in [31] for IEEE 802.11 networks.

To parameterize the ARMA filter we have simulated a scenario in which the number of terminal nodes in the network changes on every 100 seconds (the nodes were changed to 2 , $5,10,25,15,5$ and 25).

Figure 2 represents $\tilde{p}_{a}$ measured using $B=1000$ and $\alpha=0.75$. As we can see, the chosen filter parameters leads $\tilde{p}_{a}$ closer to the steady state value $p_{a}$. For a small number of nodes $\tilde{p}_{a}$ is accurately measured and the standard deviation is low. However, for a large number of nodes (e.g. in the interval 300 to $400 \mathrm{~s}$ ), $\tilde{p}_{a}$ exhibits a higher standard deviation. This degradation is mainly due to the non-linear slot busyness sampling effect, because when the number of nodes increases, the average slot duration also increases, meaning that the number of samples per time decreases (e.g. the number of samples from 300 to $400 \mathrm{~s}$ is smaller than the number of samples from 0 to $100 \mathrm{~s}$, since the average slot duration increases with the number of network nodes). This fact is also observed in [31].

Having a slowly-varying measure of $\tilde{p}_{a}$, it is possible to estimate the number of nodes applying (12). After that, we can use (11) to compute the optimal access probability. Knowing that the filter (15) introduces a slower dynamic due to the memory parameter $\alpha$, the reference $\tilde{\tau}(k)$ obtained through (11) is not directly adopted by each node. For that, we use a Proportional-Integral Controller, which is represented by $C(\tilde{\tau})$ in Fig. 1. In this way, the controller simply adapts the individual medium access probability according to the reference $\tilde{\tau}(k)$ and the ARMA filter dynamics. Figure 3 illustrates the block diagram of the PI Controller, where the Set Point is the optimal access probability computed in the previous phase.

To parameterize $K_{P}$ and $T_{I}$, the proportional gain and the integral time respectively, we simulated a scenario to study the process response to a unit step that represents a change in the number of network nodes (the nodes were changed from 0 to 25). The response of the system $\left(\tilde{p}_{a}\right)$ was measured in an open loop to evaluate the time needed to reach the steady state value. Applying the classical Ziegler-Nichols tuning methodology [32], $K_{P}$ and $T_{I}$ became approximately 0.6 and 23.81 , respectively.

Figure 4 compares the number of network nodes estimated with our approach $(\hat{n}(k))$ when the number of network nodes $(n(k))$ is changed according to the same pattern used in

Fig. 3 Proportional integral (PI) controller $C(\tilde{\tau})$

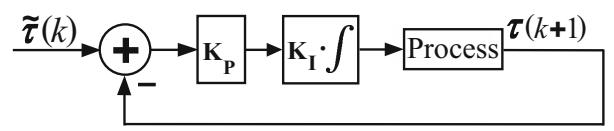




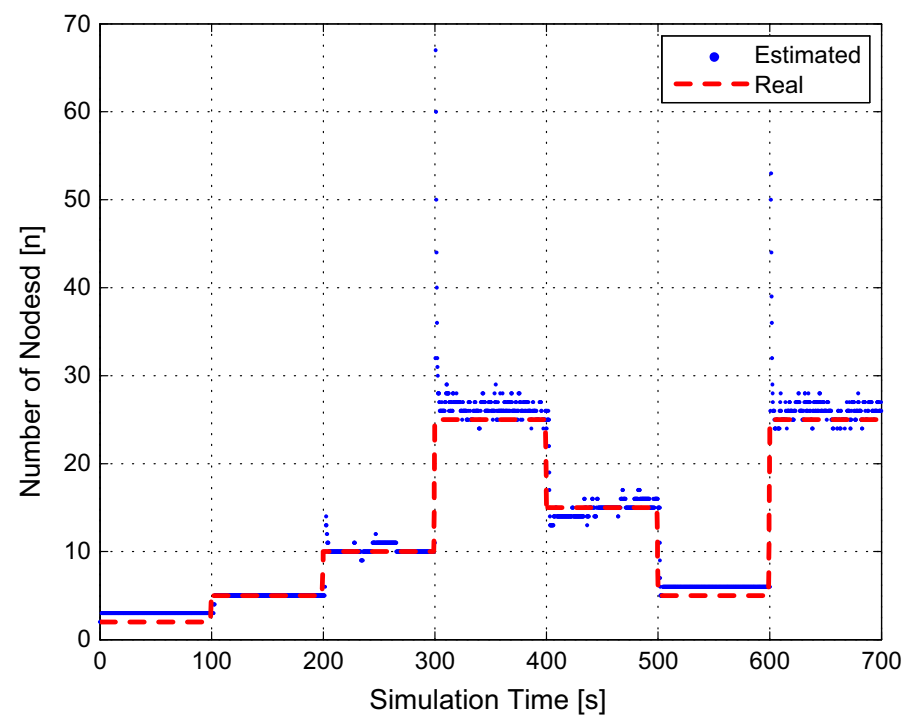

Fig. 4 Estimated number of nodes $(\hat{n})$

Fig. 2. The medium access probability is regulated according to our scheme and $W_{\text {init }}$ was set to 500. As can be observed, the estimation error increases as the number of network nodes increases. This is because the $\tilde{p}_{a}$ measurement obtained with the ARMA filter is less accurate due to the non-linear slot busyness sampling effect. When the number of nodes increases $\hat{n}$ 's accuracy decreases within a short period (peaks close to 200 and $300 \mathrm{~s}$ ), being a direct consequence of using the individual medium access probability to estimate the number of nodes. These peaks are due to the individual medium access probability, which is not yet adapted to the real number of nodes. In fact, during the peaks the individual medium access probability is higher than the optimal steady-state value for the existing number of nodes. But as the medium access probability converges to the steady state value, the estimated number of nodes becomes more accurate.

Note that MyopicMAC adapts its contention for the period $k+1$ when the contention period $k$ finishes. Moreover, since the algorithm only needs to gather information about busy and idle slots to compute (14) and (15), the transmission errors will not impact the contention regulation, because these errors only mean a failed frame decoding attempt at the receiver node. But since the frame is effectively transmitted, all nodes will detect the energy related with the frame transmission and will declare it a busy slot. This is essential to obtain a valid measurement of $\tilde{p}_{a}$, which is not influenced by the occurrence of transmission errors.

\section{Performance Analysis}

\subsection{Simulation Setup}

In this section, we present a comparative performance analysis of MyopicMAC with four other MAC algorithms: AOB [8] and Idle Sense [4], which are RA algorithms based on 
optimal contention window regulation, and the heuristic-based IEEE 802.11 [21] and GDCF [23]. The optimal throughput given by the expression (5) for the optimal medium access probability $\tau^{\star}$ was also considered. The comparison is based on simulation results. The simulations were performed with the network simulator $n s-2$ [33] by implementing a new MAC protocol that incorporates the MyopicMAC algorithm. ${ }^{3}$ We compare the MAC algorithms performance in terms of throughput, service time and channel access fairness for different number of nodes and different channel conditions (different transmission error probabilities). All nodes are traffic-saturated, i.e., each transmitting node has always a frame to transmit. The parameters used in the simulations are described in Table 1.

\subsection{Throughput and Service Time}

The optimal throughput considered in the performance analysis was computed using the expression (5), where the optimal steady-state medium access probability $\tau^{\star}$ that solves (10) is considered in $p_{i}, p_{s}$ and $p_{c}$. Once again, by adopting the throughput definition in (5), we consider that all nodes apply the same optimal medium access probability $\tau^{\star}$, and in this sense the optimal throughput means the maximum achievable throughput for a fair access policy.

The service time, also characterized in the simulation results, represents the total amount of time needed to transmit (serve) one frame, and is defined as the duration from the instant a node starts the transmission, until the instant when the transmission task effectively finishes. For a given node, the service time depends on the channel occupation due to the transmissions of its neighbors and on the number of retransmissions needed to finish the transmission task. Depending on the number of retransmissions, the service time may include one or more contention periods, one or more periods representing the effective channel occupation and the waiting time for the receiver to acknowledge the frame.

\subsection{Fairness Index}

To characterize the medium access fairness, we adopted the Jain's Index [9] as a comparative metric. Considering a given set $\mathcal{S}$ of consecutive medium accesses, the Jain's index is defined as

$$
F\left(\# \mathcal{S}, x_{i}\right)=\frac{\left(\sum_{i=1}^{n} \frac{x_{i}}{\# \mathcal{S}}\right)^{2}}{n \sum_{i=1}^{n}\left(\frac{x_{i}}{\# \mathcal{S}}\right)^{2}},
$$

where $\gamma_{i}=x_{i} / \# \mathcal{S}$ denotes the ratio of the number of medium accesses of the node $i$ in the set $\mathcal{S}$ (denoted as $x_{i}$ ) to $\mathcal{S}$ 's cardinality (represented by $\# \mathcal{S}$ ). The cardinality of $\mathcal{S}$ represents the number of consecutive medium accesses considered in the Jain's index computation, and is treated hereafter as fairness window. Perfect unfairness happens when a single node, say $n_{y}$, is responsible for all the accesses in $\mathcal{S}$, which leads to $\gamma_{y}=1$ for the node $n_{y}$ and $\gamma_{x}=0, x \neq y$, for the remaining $x$ nodes in $\mathcal{N}$. Thus, $F\left(\# \mathcal{S}, x_{i}\right)=1 / n$ for perfect unfairness. If all nodes have exactly the same number of medium accesses in $\# \mathcal{S}$ consecutive accesses (perfect fairness), this means that all nodes exhibit the same $\gamma_{i}$ ratio, and we have $F\left(\# \mathcal{S}, x_{i}\right)=1$.

3 The source code of MyopicMAC is available to download at http://tele1.dee.fct.unl.pt/downloads/miop_ MAC.tar.gz, allowing the community to evaluate their own scenarios and compare it with other algorithms. 
Table 1 Common parameters used in the simulated algorithms

\begin{tabular}{llll}
\hline SIFS & $10 \mu \mathrm{s}$ & $R$-Data rate & $11 \mathrm{Mbps}$ \\
DIFS & $50 \mu \mathrm{s}$ & Propagation delay & $2 \mu \mathrm{s}$ \\
EIFS & $364 \mu \mathrm{s}$ & $A C K$ & $416 \mu \mathrm{s}$ \\
$L_{P H Y}$ & $128 \mathrm{bits}$ & $L_{M A C}$ & $224 \mathrm{bits}$ \\
Slot time & $20 \mu \mathrm{s}$ & $B$ - Sampling window size & 1000 \\
$W_{\text {init }}$ & 500 & $L_{D A T A}$ & 1500 bytes \\
$K_{P}$ & 0.6 & $T_{I}$ & 23.81 \\
\hline
\end{tabular}

The fairness window $\# \mathcal{S}$ also plays an important role in Jain's index. If $\# \mathcal{S}$ is too small, this means that we analyze the number of accesses per node $\left(x_{i}\right)$ for a short number of accesses $(\# \mathcal{S})$. This case represents a short-term analysis, and the probability of achieving perfect fairness $\left(F\left(\# \mathcal{S}, x_{i}\right)=1\right)$ in short-term is low because the probability of having the same number of medium accesses per node in a short sample of length $\# \mathcal{S}$ is also low. But as the fairness window increases, more accesses are considered in the index computation. This case represents a long-term analysis, and it is expected that the Jain's index converges to 1 as $\# \mathcal{S}$ converges to infinity [9]. For the Jain's index results presented in the next subsection we have considered a scenario of 24 nodes $(n=24)$ and different fairness window values, which characterize the short, medium and long-term fairness.

It is worth highlighting the importance of the medium access fairness in ad hoc networks. This is mainly because the performance of the upper layer protocols substantially rely on the MAC fairness. Low fairness means an increase in the variance of the time needed to transmit a frame, since some nodes will wait longer to transmit. This effect decreases the performance of several massively used protocols. Specific reports can be found for: TCP [34]; or for real-time communications (voice, video, etc.), where the variance usually results in an unbounded jitter [35]. These works show that most of the times the throughput is not the most important point to mention in distributed wireless networks, since the protocols adopted in the upper layers only present high performance when the MAC schemes are almost perfectly fair.

\subsection{Simulation Results}

This subsection compares the throughput, service time and fairness achieved by each protocol and for different number of nodes and channel conditions.

Figure 5 illustrates the results obtained when the transmission errors in the physical layer are almost negligible (FER $=10^{-4}$ ). The Jain's fairness index was computed for different fairness windows, ranging from $\# \mathcal{S}=25$ (the number of transmitting users) up to $\# \mathcal{S}=1500$ medium accesses. Therefore, short-term and long-term fairness is analyzed. Figure $5 \mathrm{c}$ indicates that our approach presents the highest fairness index for the fairness window sizes considered in the analysis. This is because MyopicMAC approach imposes a similiar medium access probability $(\tau)$ to all network nodes, which is close to the optimal value. From the throughput results shown in Fig. 5(a) we observe that most of the MAC algorithms present throughput values that are higher than the fairness optimal one. This is due to the optimal throughput definition [Eq. (5) assuming $\tau^{\star}$ in (10)], which assumes perfect fairness (all nodes adopt the same $\tau^{\star}$ probability). If the medium access is not fair, some nodes can have shorter contention periods, and the network throughput can be higher than the optimal value. In the limit, if a single node adopts the maximum medium access probability (equal to 1) and the remaining nodes inhibit their access, the throughput equals 

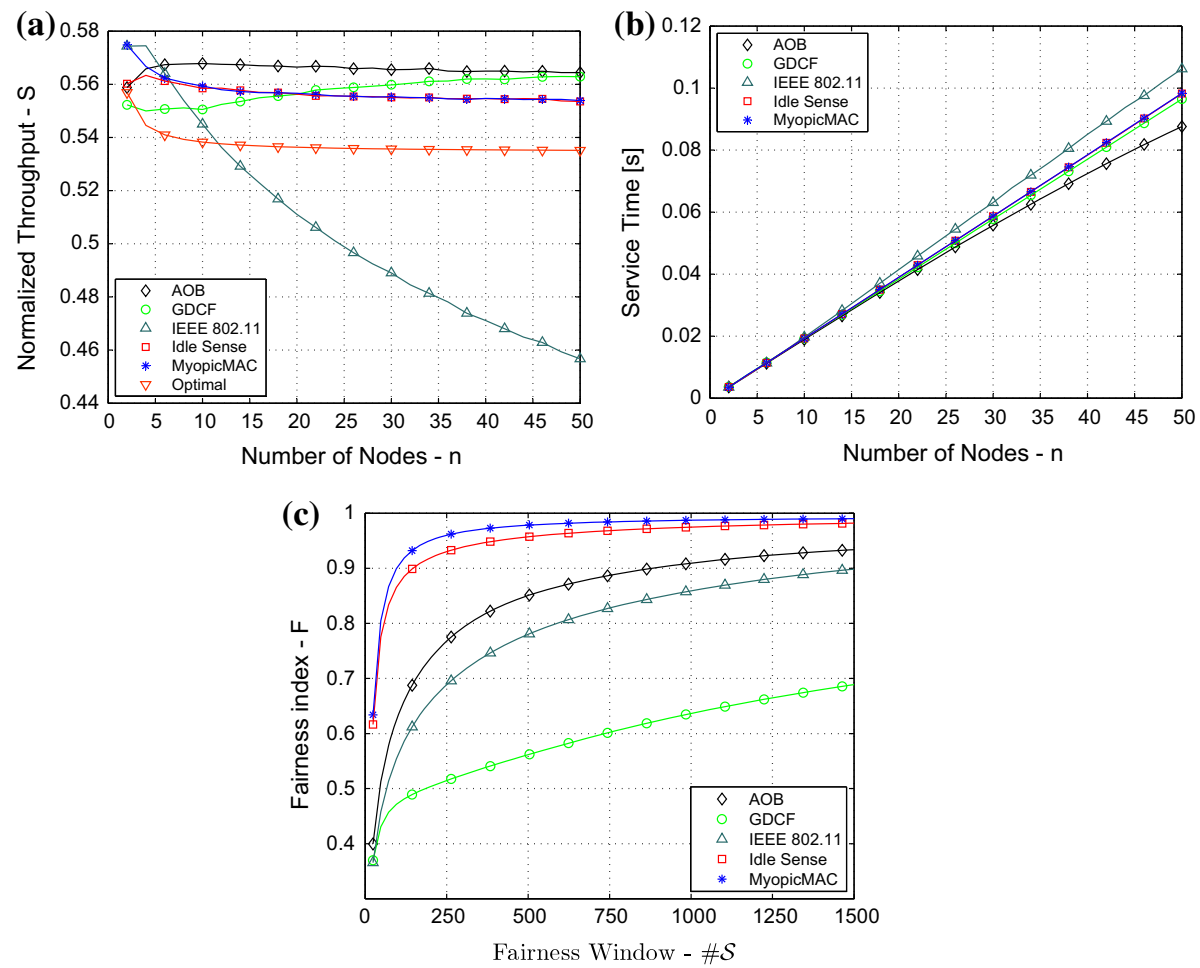

Fig. 5 Average throughput, service time and Jain's fairness index for $E_{B} / N_{0}=15.5 \mathrm{~dB} \Rightarrow p_{e} \approx 10^{-4}$. a Average throughput. b Average service time. c Jain's index for 24 nodes

the maximum network usage $(S=1)$, which outperforms the optimal throughput for perfect fair medium access. As the number of nodes increase, we observe that MyopicMAC presents a throughput performance close to IS, and slightly below AOB. Considering the specific case when the network is formed by 24 nodes (because the Jain index is plotted for this case), AOB, IS and GDCF present higher throughput than the optimal one, denoting that they are not perfectly fair. This can be observed in Fig. 5c, where GDCF exhibits lower access fairness, followed by AOB, which is also followed by IS. Because the optimal throughput assumes perfect fairness, a deviation from the optimal throughput means a decrease in terms of medium access fairness. Note that MyopicMAC achieves almost the same throughput as IS, but because it is slightly closer to the optimal throughput, it outperforms IS in terms of medium access fairness.

In terms of average service time, all protocols have a similar performance when the number of nodes is up to 20 . Moreover, all protocols present a service time that grows linearly with the number of nodes, except AOB, which behaves better. This is mainly because the approximation used in AOB postpones the channel access when the probability of successful transmission is low, which decreases the time wasted in unsuccessful transmission attempts. Moreover, note that the transmission queue waiting time is not accounted in the service time. Since AOB exhibits low fairness, the frame waiting time, which incorporates the service time and the queue's waiting time, presents higher variance. This means that the nodes accessing less frequently the channel will exhibit higher frame 
(a)

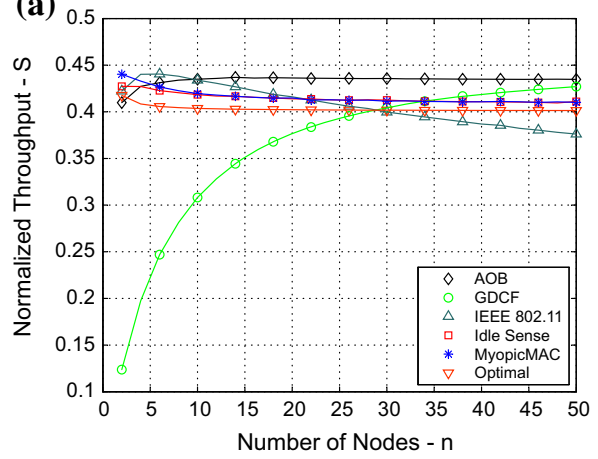

(b) 0.

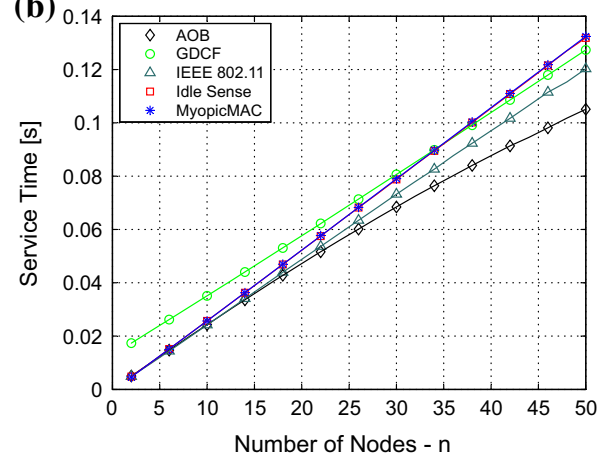

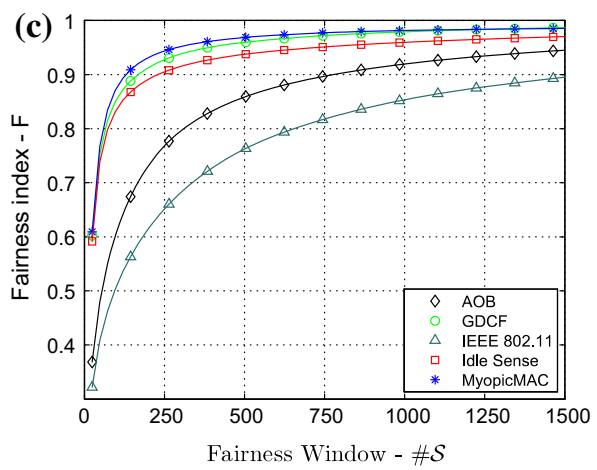

Fig. 6 Average throughput, service time and Jain's fairness index for $E_{B} / N_{0}=13.0 \mathrm{~dB} \Rightarrow \mathrm{p}_{\mathrm{e}} \approx 0.25$. a Average throughput. b Average service time. c Jain's index for 24 nodes

access times than the nodes accessing more frequently, which penalizes some frames transmitted by the AOB protocol. Moreover, it is well known that this fact decreases the performance of the upper protocols, as reported in [34] and [35].

The performance of the IEEE 802.11's throughput clearly shows that this protocol is highly dependent on the number of nodes. The throughput decays with the number of nodes. When compared to IEEE 802.11, the heuristic-based GDCF protocol does not present the problem associated with the throughput decay, but it underperforms in terms of medium access fairness. This observation evidences the superiority of the optimal-based protocols, mainly MyopicMAC and IS, when the FER is low.

Figure 6 presents the results for a FER=0.25. As can be observed, GDCF exhibits low throughput when the number of nodes is approximately smaller than 30 nodes. This is because a high amount of unacknowledged frames due to transmission errors increases the period of contention to undesired levels, which substantially decreases the throughput and improves the access fairness. This can be clearly observed by comparing GDCF's throughput depicted in Fig. 6a with the case illustrated in Fig. 5a. GDCF's higher service time can be also observed in Fig. 6b, namely for a number of nodes below 30. Regarding MyopicMAC and IS protocols, Fig. 6a shows that its throughput is not affected by the physical errors, since the throughput decrease is proportional to the FER. In terms of medium access fairness, MyopicMAC and IS still present high performance. Note that for this case $(\mathrm{FER}=0.25)$, GDCF is frequently applying its maximum contention window, 
which means that all nodes converge to the same medium access probability. However, the throughput illustrated in Fig. 6a does not recommend GDCF's utilization when transmission errors exist.

IS and MyopicMAC behave well when transmission errors exist. Contrarily, IEEE 802.11, AOB and GDCF suffer higher deviation from the optimal throughput or lower access fairness, because they regulate the contention window based on the success of the previous transmitted frame. The results show that the optimal-based protocols behave better, especially IS and MyopicMAC, since they do not suffer the influence of the transmission errors.

In a nutshell, the comparative results presented in Figs. 5 and 6 evidence the underperformance of heuristic-based protocols, namely because they use the transmission success information to regulate the contention window, being dependent on transmission errors. The optimal protocol AOB, which also uses information about the transmission success, performs better than the analyzed heuristic-based protocols. Finally, the comparative analysis clearly shows that IS and MyopicMAC outperform the other analyzed protocols in terms of the impact of transmission errors. This is mainly because no information about the success of the previous transmission is used to regulate the contention window. Moreover, the results show that MyopicMAC exhibits a higher Jain index for all fairness windows considered, showing that it performs better than IS in terms of medium access fairness. This characteristic is important because it increases the performance of the communication stack, as reported in [34, 35].

\subsection{MyopicMAC's Response Time}

This subsection analyzes MyopicMAC's response time, which indicates how quickly the contention window will react to changes on the number of nodes. Figure 7 illustrates the contention window regulation when the number of nodes competing for the medium increases from 5 to 10 (Fig. 7a) and from 5 to 25 (Fig. 7b). In both cases, 5 or 20 nodes start transmitting at time instant $5.0 \mathrm{~s}$, respectively.

The red curve presents the optimal contention window (reference) for the number of active nodes. As can be observed, the time needed to cross the reference is approximately 1 and $2 \mathrm{~s}$ when 5 and 20 nodes start transmitting, respectively. The response time is shorter for the case when a smaller number of nodes start transmitting. As more nodes start transmitting, the expected duration of the slots increase, which means that the ARMA filter takes more time to sample the $B$ slots and, consequently, the measurement of the probability of finding an idle slot $\left(\tilde{p}_{a}\right)$ takes a longer time to update. Therefore, the contention window takes a longer time to converge to the optimal one, justifying the higher response time for the case when 20 nodes start transmitting (Fig. 7b).

To better analyze the impact of the response time, we can roughly define an upper bound of the number of transmissions needed to reach the optimal point of operation in a perfect fair MAC scheme. Considering a MAC scheme with a service time denoted by $T_{S}$, $n$ nodes will take $n \times T_{S}$ seconds to transmit. Representing the time needed to reach the optimal point of operation by $T_{O}$, the upper bound of the number of transmissions needed to reach the optimal point of operation is given by

$$
\mathcal{O}(\Gamma) \approx \frac{T_{O}}{n T_{S}}
$$



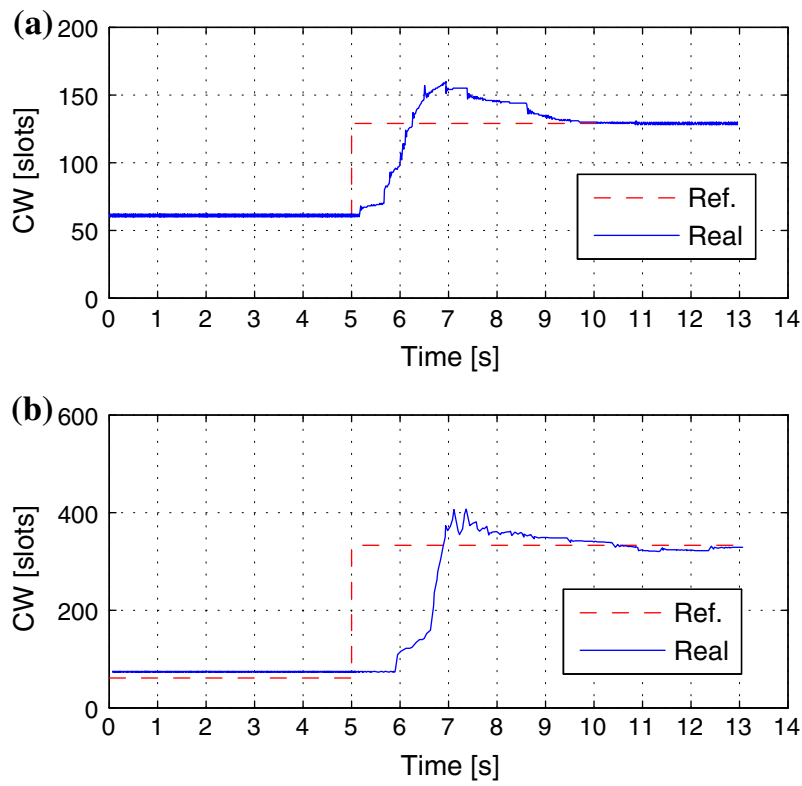

Fig. 7 Response time of the contention window regulation when the number of nodes increases at time instant 5.0 s from: a 5 nodes to 10 nodes; b 5 nodes to 25 nodes

By observing Fig. $5 \mathrm{~b}$, the service time, $T_{S}$, for a number of nodes equal to 5 is approximately $0.012 \mathrm{~s}$. If the response time, $T_{O}$, for a change from 5 to 10 active nodes is approximately $1.0 \mathrm{~s}$, the upper bound of the number of transmissions needed to reach the optimal point of operation is approximately 9 transmissions $(1.0 \mathrm{~s} /(0.012 \mathrm{~s} \times 1$ nodes $))$. Following the same rationale, and considering an average service time of $0.037 \mathrm{~s}$ for a number of nodes between 5 and 25, each node operates near the optimal point of operation after the third transmission $(2.0 \mathrm{~s} /(0.037 \mathrm{~s} \times 25$ nodes $))$. Analyzing the scenario of nonsaturated traffic, where the nodes can generate a finite burst of frames, the previous results show two important points:

- For a small number of nodes accessing the medium, each node needs to perform more transmissions before having its contention window near the optimal point of operation. This is a reasonable behavior, since the optimal contention window will be in the same order of magnitude of the present one. However, for a short burst of frames (lower than 9 frames in the case depicted in Fig. 7a), the contention window may never reach the optimal reference;

- For a large number of nodes accessing the medium, the contention window will take more time to be adapted. But since the service time per frame also increases, this means that a short burst of frames will take longer time to be served, and the contention window will be adapted within a smaller number of transmissions (nine for the scenario considered in Fig. 7a and only three in the scenario considered in Fig. 7b).

Considering the observations described above, and despite MyopicMAC's design being based in traffic-saturated nodes, it tolerates non-saturated nodes. Moreover, MyopicMAC's performance only depends on the length of the bursts and number of nodes generating it. In 
other words, for a small number of nodes, if the bursts of frames are short, the nodes may never reach the optimal point of operation, and MyopicMAC may underperform. If the number of nodes generating bursty traffic increases, the optimal point of operation can be reached even for short bursts, because the service time and the transmission queueing delay increases, and the non-saturated generation converges to a scenario equivalent to the saturated one, where each node has always a frame to transmit.

\subsection{Final Remarks}

While assuming that transmission errors may occur (due to several effects occurring in the PHY-layer), we highlight that in MyopicMAC the contention window regulation does not depend on the success of the previous transmission attempt. This means that the behavior of MAC scheme adopted in MyopicMAC is not affected by transmission errors. As we can see from Eqs. (10) and (11) derived in Sect. 4.1, the optimal contention window only depends on the number of nodes competing for the wireless channel. The number of nodes competing for the wireless channel is estimated using (14) in (15) and (15) in (12), and the estimation only needs information about the slots sensed idle or busy to compute (14). Consequently, whenever a transmission is attempted, the competing nodes will detect the energy related with the frame transmission and will observe a busy slot independently of the success of the transmission. In this way, and contrarily to the MAC scheme adopted in IEEE 802.11, the transmission errors at the PHY-layer do not influence MyopicMAC's contention window regulation.

\section{Conclusions}

In this paper, we proposed the MyopicMAC algorithm, a distributed throughput optimal MAC scheme for single-hop distributed wireless networks. The algorithm is mainly motivated by the shared view of the channel. The main novelty of our scheme is the use of the individual medium access probability and the probability of idle slot to estimate the number of competing nodes. Several simulations show that MyopicMAC successfully adapts to the number of the network nodes. Since each node shares the same channel view, and because the contention window mainly depends on it, the simulation results confirm that our scheme achieves better access fairness meaning that its average throughput is closer to the optimal curve. Finally, because MyopicMAC does not take the success of previous transmissions in the contention regulation, the results show that the performance of the MAC algorithm is not affected by physical transmission errors.

Our approach is a first step towards devising more sophisticated algorithms for decentralised medium access control without requiring message passing. Future research will focus on a solution for a multi-hop scenario, where the MAC reliability must be improved to cover the problem associated with the hidden/exposed terminals.

Acknowledgments This work was supported by FCT/MEC-funded projects MANY2COMWIN (EXPL/ EEI-TEL/0969/2013), ADIN (PTDC/EEI-TEL/2990 /2012) and UID/EEA/50008/2013. Part of this work was also supported by the grant SFRH/BD/68367/2010. 


\section{Appendix}

In this appendix we derive (10). Assuming that the expected durations $\mathbb{E}\left[L_{D A T A} / R\right], \mathbb{E}\left[T_{s}\right]$, $\mathbb{E}\left[T_{i}\right], \mathbb{E}\left[T_{c}\right]$ can be constants denoted by, $D, T_{s}, T_{i}$ and $T_{c}$, respectively, and using (4), (5) can be rewritten as

$$
\begin{aligned}
S & =\frac{p_{s}\left(1-p_{e}\right) \mathbb{E}\left[L_{D A T A} / R\right]}{p_{i} \mathbb{E}\left[T_{i}\right]+p_{s} \mathbb{E}\left[T_{s}\right]+p_{c} \mathbb{E}\left[T_{c}\right]} \\
= & \frac{p_{s}\left(1-p_{e}\right) D}{p_{i} T_{i}+p_{s} T_{s}+p_{c} T_{c}} \\
= & \frac{\left(1-p_{e}\right) D}{T_{s}-T_{c}+\frac{T_{c}+p_{i}\left(T_{i}-T_{c}\right)}{p_{s}}} .
\end{aligned}
$$

Using (2) and (3), $S$ is rewritten as

$$
S=\frac{\left(1-p_{e}\right) D}{T_{s}-T_{c}+\frac{T_{c}+(1-\tau)^{n}\left(T_{i}-T_{c}\right)}{n \tau(1-\tau)^{n-1}}} .
$$

Since $p_{e}$ and $\tau$ are independent variables and because $D, T_{s}$ and $T_{c}$ are constants, to maximize $S$ by adopting the appropriate medium access probability $\tau$ we need to maximize the following quantity

$$
\chi(\tau)=\frac{n \tau(1-\tau)^{n-1}}{T_{c}+(1-\tau)^{n}\left(T_{i}-T_{c}\right)} .
$$

$\chi$ is maximized by solving $\frac{\partial \chi}{\partial \tau}=0$, and

$$
\frac{\partial \chi}{\partial \tau}=\frac{n(1-\tau)^{n-1}\left[(1-\tau)^{n}\left(T_{c}-T_{i}\right)+(n \tau-1) T_{c}\right]}{(\tau-1)\left[(1-\tau)^{n}\left(T_{c}-T_{i}\right)-T_{c}\right]^{2}} .
$$

Finally, the throughput $S$ is maximized by finding the access probability $0<\tau<1$ that solves $\frac{\partial \chi}{\partial \tau}=0$, and the optimal access probability $\tau^{\star}$ is given by

$$
\left(1-\tau^{\star}\right)^{n}\left(T_{c}-T_{i}\right)+\left(n \tau^{\star}-1\right) T_{c}=0 .
$$

\section{References}

1. Shah, D., Tse, D. N. C., \& Tsitsiklis, J. N. (2011). Hardness of low delay network scheduling. IEEE Transactions on Information Theory, 57(12), 7810-7817.

2. Bianchi, G., Fratta, L., \& Oliveri, M. (1996) Performance evaluation and enhancement of the CSMA/ CA MAC protocol for 802.11 wireless LANs. In Personal, indoor and mobile radio communications, 1996. PIMRC'96., seventh IEEE international symposium on, pp. 392-396.

3. Rad, A. H. M., Huang, J., Chiang, M., \& Wong, V. W. S. (2009). Utility-optimal random access without message passing. IEEE Transactions on Wireless Communications, 8(3), 1276-1536. 
4. Heusse, M., Rousseau, F., Guillier, R., \& Duda, A. (2005). Idle sense: An optimal access method for high throughput and fairness in rate diverse wireless LANs. Sigcomm Computer Communication Review Journal, 35(4), 121-132. doi:10.1145/1090191.1080107

5. Garcia-Saavedra, A., Banchs, A., Serrano, P., \& Widmer, J. (2012). Distributed opportunistic scheduling: A control theoretic approach. In INFOCOM, 2012 Proceedings IEEE, pp. 540-548.

6. Leith, D. J., Qizhi Cao, C., \& Subramanian, V. G. (2012). Max-min fairness in 802.11 mesh networks. IEEE/ACM Transactions on Networking, 20(3), 756-769.

7. Patras, P., Banchs, A., Serrano, P., \& Azcorra, A. (2011). A control-theoretic approach to distributed optimal configuration of 802.11 WLANs. IEEE Transactions on Mobile Computing, 10(6), 897-910.

8. Bononi, L., Conti, M., \& Gregori, E. (2004). Runtime optimization of IEEE 802.11 wireless LANs performance. IEEE Transactions on Parallel and Distributed Systems, 15(1), 66-80.

9. Jain, R. K., Chiu, D. -M., \& Hawe, W. R. ( Sept. 1984). A quantitative measure of fairness and discrimination for resource allocation in shared computer systems. DEC Research, DEC-TR-301.

10. Abramson, N. (1970). The ALOHA system: Another alternative for computer communications. In Proceedings of the November 17-19, 1970, Fall Joint Computer Conference (pp. 281-285). AFIPS '70 (Fall). New York: ACM.

11. Lee, J.-W., Tang, A., Huang, J., Chiang, M., \& Calderbank, A. R. (2007). Reverse-engineering MAC: A non-cooperative game model. Selected Areas in Communications, IEEE Journal on, 25(6), 1135-1147. doi:10.1109/JSAC.2007.070808

12. Joo, C., \& Shroff, N. B. (Nov. 2006). Performance of random access scheduling schemes in multi-hop wireless networks. In Proceedings of ACSSC, pp. 1937-1941.

13. Chen, L., Low, S. H., \& Doyle, J. C. (March 2005). Joint congestion control and media access control design for ad hoc wireless networks. In INFOCOM 2005. 24th Annual Joint Conference of the IEEE Computer and Communications Societies. Proceedings IEEE, Vol. 3, pp. 2212-2222.

14. Lee, J.-W., Chiang, M., \& Calderbank, A. R. (2007). Utility-optimal random-access control. IEEE Transactions on Wireless Communications, 6(7), 2741-2751.

15. Oliveira, R., Bernardo, L., \& Luis, M. (March 2011). Analysis of Heuristic-based MAC protocols for ad hoc Networks. In Proceedinfs of IEEE WCNC, pp. 191-196.

16. Chiang, M., Low, S. H., Calderbank, A. R., \& Doyle, J. C. (2007). Layering as optimization decomposition: A mathematical theory of network architectures. Proceedings of the IEEE, 95(1), 255-312. doi:10.1109/JPROC.2006.887322

17. Tassiulas, L., \& Ephremides, A. (1992). Stability properties of constrained queueing systems and scheduling policies for maximum throughput in multihop radio networks. IEEE Transactions on Automatic Control, 37(12), 1936-1948.

18. Rajagopalan, S., \& Shah, D. (March 2008). Distributed algorithm and reversible network. In Information Sciences and Systems, 2008. CISS 2008. 42nd Annual Conference on, pp. 498-502.

19. Modiano, E., Shah, D., \& Zussman, G. (2006). Maximizing throughput in wireless networks via gossiping. In Proceedings of the Joint International Conference on Measurement and Modeling of Computer Systems (pp. 27-38). SIGMETRICS '06/Performance '06. New York: ACM.

20. Shakkottai, S., \& Srikant, R. (2007). Network optimization and control. Foundations and Trends in Networking, Now Publishers, 2(3), 271-379.

21. ANSI/IEEE 802.11 Standard. (2007). IEEE Standard for Information technology-Telecommunications and information exchange between systems-Local and metropolitan area networks-Specific requirements-Part 11: Wireless LAN medium access control (MAC) and physical layer (PHY) specifications. 2007.

22. Jurdak, R., Lopes, C. V., \& Baldi, P. (2004). A survey, classification and comparative analysis of medium access control protocols for ad hoc networks. IEEE Communications Surveys \& Tutorials, 6(1), 2-16. First Quarter.

23. Wang, C., Li, B., \& Li, L. (2004). A new collision resolution mechanism to enhance the performance of IEEE 802.11 DCF. IEEE Transactions on Vehicular Technology, 53(4), 1235-1246.

24. Kwon, Y., Fang, Y., \& Latchman, H. (April 2003). A Novel MAC Protocol with Fast Collision Resolution for Wireless LANs. In INFOCOM 2003. Twenty-Second Annual Joint Conference of the IEEE Computer and Communications. IEEE Societies, Vol. 2, pp. 853-862.

25. Shin, J. (Sept. 2010). Efficient distributed medium access algorithm. Ph.D. thesis, Massachusetts Institute of Technology.

26. Bianchi, G. (2000). Performance analysis of the IEEE 802.11 distributed coordination function. IEEE Journal on Selected Areas in Communications, 18(3), 535-547.

27. Bertsekas, D., \& Gallager, R. (1991). Data networks. New Jersey: Prentice-Hall.

28. Proakis, J. G., \& Salehi, M. (2007). Digital communications. New York: McGraw-Hill. 
29. Kumar, P. R., \& Meyn, S. P. (1995). Stability of queueing networks and scheduling policies. IEEE Transactions on Automatic Control, 40(2), 251-260.

30. Georgiadis, L., Neely, M. J., \& Tassiulas, L. (2006). Resource allocation and cross-layer control in wireless networks. Foundations and Trends in Networking, Now publishers, 1(1), 1-149.

31. Bianchi, G., \& Tinnirello, I. (2003). Kalman filter estimation of the number of competing terminals in an IEEE 802.11 network. In INFOCOM 2003. Twenty-Second Annual Joint Conference of the IEEE Computer and Communications. IEEE Societies, Vol. 2, pp. 844-852, March-April 2003.

32. Kuo, B. C., \& Golnaraghi, F. (2002). Automatic control systems. New York: Wiley.

33. Information Sciences Institute. NS-2 Network Simulator (version 2.33). Software Package retrieved from http://www.isi.edu/nsnam/ns/, 2010.

34. Xu, K., Gerla, M., Qi, L., \& Shu, Y. (2003). Enhancing TCP fairness in ad hoc wireless networks using neighborhood RED. In Proceedings of the 9th Annual International Conference on Mobile Computing and Networking, pp. 16-28. MobiCom '03. New York: ACM.

35. Wang, P., Jiang, H., \& Zhuang, W. (2008). A New MAC scheme supporting voice/data traffic in wireless Ad Hoc networks. IEEE Transactions on Mobile Computing, 7(12), 1491-1503.

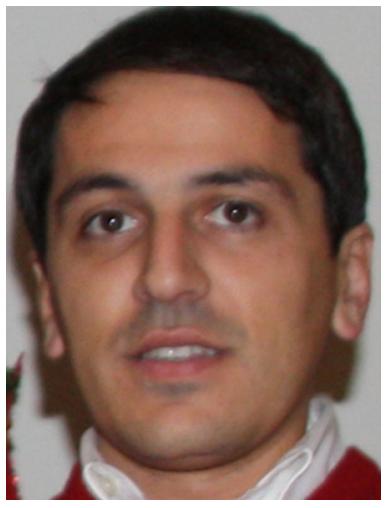

Rodolfo Oliveira obtained the Licenciatura degree in Electrical Engineering from Universidade Nova de Lisboa, Lisbon, Portugal in 2000 and the M.Sc. degree in Electrical and Computer Engineering from Technical University of Lisbon in 2003. In 2009, he received the $\mathrm{Ph} . \mathrm{D}$ degree in Electrical Engineering from Universidade Nova de Lisboa, where he is currently Assistant Professor at the Department of Electrotechnical Engineering and is also affiliated as a researcher with the Centre of Technology and Systems (CTS), Uninova. From October 2007 to March 2008 he was an Invited Researcher at University of Thessaly, Greece, where he worked with Prof. Leandros Tassiulas. Rodolfo Oliveira published approximately fifty papers in Journals and international conferences, and participated in a dozen of national and international research projects. Rodolfo Oliveira was invited by Carneggie Mellon University (CMU), Pittsburgh, USA, to be an Invited Scholar from August 2011 to December 2011. His interests lie in the area of medium access control for wireless mobile systems, network modeling and performance analysis, and design of cross-layer protocols for mobile unstructured networks, with applications in ad hoc, vehicular and sensor networks. Rodolfo Oliveira is a member of the IEEE and the ACM.

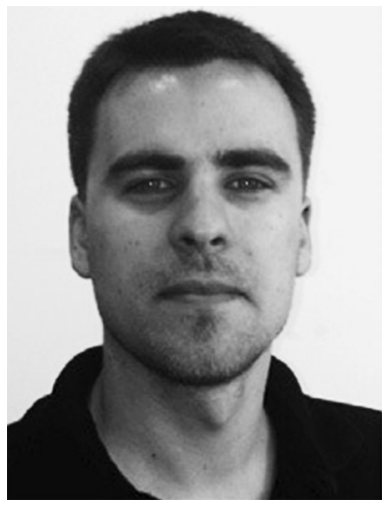

Miguel Luis recevied the M.Sc. degree in electrical engineering and computers from the Faculdade de Ciências e Tecnologia, Universidade Nova de Lisboa (FCT-UNL), Portugal in 2009. In the beginning of 2010 he joined the research center Instituto de Desenvolvimento de Novas Tecnologias (UNINOVA), and later the Instituto de Telecomunicações (IT) where he has been involved in several research projects concerning wireless systems. Currently, he is a Ph.D. student at FCT-UNL, supported by a grant from Fundação para a Ciência e Tecnologia (FCT-MEC) since 2011. His main research interests include medium access control protocols for wireless systems, particularly cognitive radio networks. 

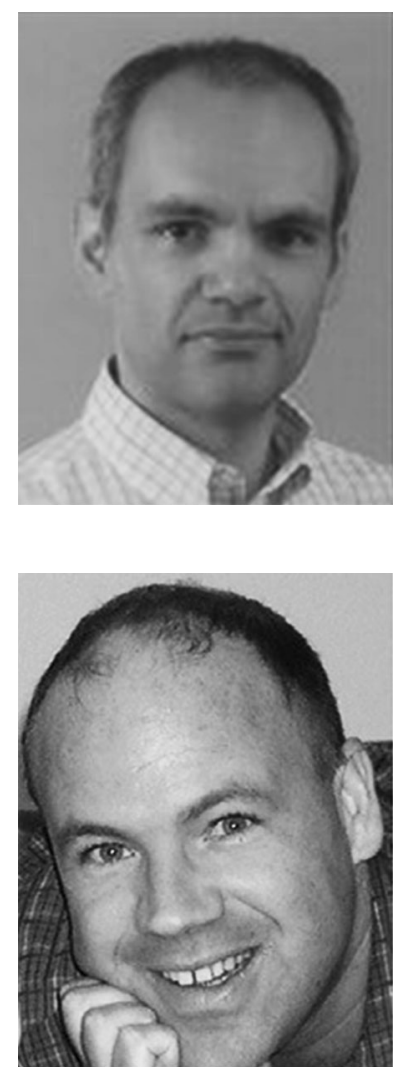

Luis Bernardo received the Ph.D. degree in Electrical Engineering and Computers from Instituto Superior Técnico (IST), Technical University of Lisbon, Portugal, in 2002. He is an Assistant Professor at FCT-UNL (Faculdade de Ciências e Tecnologia da Universidade Nova de Lisboa) since 1999. Since 2000 he is a researcher at Instituto de Desenvolvimento de Novas Tecnologias (UNINOVA), and in 2013 he joined the research center IT (Instituto de Telecomunicações). His main research interests include MAC protocols for wireless communications, routing protocols and network modeling.

Rui Dinis received the Ph.D. degree from Instituto Superior Técnico (IST), Technical University of Lisbon, Portugal, in 2001. From 2001 to 2008 he was a Professor at IST. Since 2008 he is teaching at FCT-UNL (Faculdade de Ciências e Tecnologia da Universidade Nova de Lisboa). He was a researcher at CAPS/IST (Centro de Análise e Processamento de Sinais) from 1992 to 2005; from 2005 to 2008 he was researcher at ISR/IST (Instituto de Sistemas e Robótica); in 2009 he joined the research center IT (Instituto de Telecomunicações). He has been involved in several research projects in the broadband wireless communications area. His main research interests include modulation, equalization, synchronization and channel estimation. 